\title{
GÁS DISSULFETO DE CARBONO NA QUEBRA DE DORMÊNCIA DA BATATA-SEMENTE
}

\author{
CARBON DISSULFATE GAS IN THE BREAK OF DORMANCY \\ OF THE POTATO-SEED
}

\author{
Stela Maris de Oliveira KOWALSKI \\ Edilberto POSSAMAI ${ }^{2}$ \\ João Carlos POSSAMAl ${ }^{3}$ \\ Francisco Antonio MARÇALLO ${ }^{4}$
}

\begin{abstract}
RESUMO
O objetivo desta pesquisa foi o de avaliar a eficiência do gás dissulfeto de carbono em diferentes doses e tempo de aplicação na quebra de dormência de tubérculos de 3 cultivares de batata-semente, apos 30 dias da colheita. $O$ experimento foi conduzido no Laboratório de Sementes do Departamento de Fiitotecnia e Fitossanitarismo do Setor de Ciências Agrárias da UFPR. Foram utilizados tubérculos de batata-semente do tipo II (entre 41 e $50 \mathrm{~mm}$ de diâmetro). Foram realizados dois experimentos: $1^{\text {a }}$ - Outubro de 2003 com as cultivares: Asterix e Cupido de Guarapuava - PR e a $2^{\mathrm{a}}$ - Fevereiro de $2004 \mathrm{com}$ as cultivares: Asterix e Monalisa de Canoinhas-SC. Os tratamentos foram exposições das batatas-sementes com o gás disssufeto de carbono nas doses: 1,04, 1,38, 1,73, 2,07 e 2,42 ml.cm ${ }^{-3}$ durante os períodos de 60,72 e 84 horas. Os tubérculos foram acondicionadas em caixas de papelão $\left(69,120 \mathrm{~cm}^{3}\right)$. Cada caixa com 20 tubérculos. O gás dissulfeto de carbono foi colocado em um pote plástico $(250 \mathrm{ml})$. Em seguida as caixas foram vedadas com fita adesiva e colocadas dentro de um saco plástico de polietileno para evitar a evaporação do gás. As batatas-sementes foram mantidas há uma temperatura de $22^{\circ} \mathrm{C}$ a 25 $\mathrm{C}$ sob luz indireta, durante os 30 dias de avaliação. As leituras foram realizadas semanalmente totalizando três (3) leituras de contagem do número de brotos por tubérculos e a determinação do comprimento de cada broto. O delineamento foi inteiramente casualizado, no esquema fatorial (3x5) com quatro (4) repetições. A separação das médias foi determinada pelo DMS (99\%). Os resultados do experimento 01, para as cultivares Asterix e Cupido, sugerem que o uso do gás dissulfeto de carbono é eficaz para o rompimento da dormência, promovendo maior número e comprimento de brotos quando os tubérculos são expostos ao gás no tempo de 72 horas na dosagem de $1,73 \mathrm{ml}_{\mathrm{cm}}{ }^{-3}$. Experimento 02, para as cultivar Asterix e Monalisa, os resultados sugerem o uso do gás dissulfeto de carbono no tempo de exposição de 72 horas. As dosagens para este experimento não foram significativos.
\end{abstract}

Palavras-chave: brotação, tratamento químico, Solanum tuberosum L.

\begin{abstract}
The objective of this research was to evaluate the efficiency of the carbon dissulfate gas in different doses and time of application in breaking the dormancy of tubercles of 3 cultivars of potato-seed, after 30 days of harvesting. The experiment was conducted in the Seed Laboratory of the Department of Fitotecnia and Fitossanitarismo of the Section of Agrarian Sciences of UFPR. Was used tubercle of potato-seed of the type II (between 41 and $50 \mathrm{~mm}$ diameter). The research work was divided in two stages: 1st - October of 2003 with the cultivars: Asterix and Cupido from Guarapuava - PR; and 2nd - February of 2004 with the cultivars: Asterix and Monalisa from Canoinhas-SC. The treatments were exposing the potato-seeds with the carbon dissulfate gas in the doses: 1,04, 1,38, 1,73, 2,07 and $2,42 \mathrm{ml} \cdot \mathrm{m}^{-3}$ during the periods of 60,72 and 84 hours. The tubercle were conditioned in carton boxes $\left(69,120 \mathrm{~cm}^{3}\right)$. Each carton box with 20 tubercle. The carbon dissulfate gas was placed in a plastic tray $(250 \mathrm{ml})$. Soon after the carton boxes were sealed with adhesive tape and placed inside of a plastic bag polyethylene to avoid the evaporation of the gas. The potato-seeds were maintained in the temperature of $22^{\circ} \mathrm{C}$ up to $25^{\circ} \mathrm{C}$ under indirect light, during the 30 days of evaluation. The data were weekly performed totalizing three readings of the number of sprouts by tubercle and the determination of the length of each sprout. The experimental design was a completely randomized design, in a factorial experiment (3x5) with four (4) replications. The mean separation was determined by DMS (99\%). The results of the experiment 01, for the cultivars Asterix and Cupido, suggest that the use of the carbon dissulfate gas is effective for breaking the dormancy, promoting larger number and length of sprouts when the tubercles were exposed to the gas in the time of 72 hours in the dose of $1,73 \mathrm{ml}$. $\mathrm{cm}^{-3}$. The experiment 02, for cultivars Asterix and Monalisa, the results suggest the use of the carbon dissulfate gas in the time of exhibition of 72 hours. The doses for this experiment were not significant.
\end{abstract}

Key-words: sprouting, chemical treatment, Solanum tuberosum L.

${ }^{1}$ Eng. Agrônoma, Mestranda UFPR (stela@ambienteimagem.com.br);

${ }^{2}$ Engenheiro Agrônomo, Ph. D, Professor Adjunto do Departamento de Fitotecnia e Fitossanitarismo do Setor de Ciências Agrárias da Universidade Federal do Paraná;

${ }^{3}$ Engenheiro Agrônomo, M.Sc., Professor Adjunto do Departamento de Fitotecnia e Fitossanitarismo do Setor de Ciências Agrárias da Universidade Federal do Paraná;

${ }^{4}$ Engenheiro Agrônomo, M.Sc. Professor Substituto do Departamento de Fitotecnia e Fitossanitarismo do Setor de Ciências Agrárias da Universidade Federal do Paraná. 


\section{INTRODUÇÃO}

O plantio de batata (Solanum tuberosum L.) no Brasil, teve início no século $\mathrm{XX}$, e somente na década de 70 ocorreu a maior expressão na produção de batata-semente no país. Atualmente, a cultura ocupa uma área de 171 mil hectares com produção média de 2,6 milhões de toneladas a taxa de utilização de batata-semente melhorada (básica, registrada e certificada) no país é de 25 a $30 \%$ com produção nacional de 64.000 a 96.000 toneladas de batatasemente para uma demanda de 420.000 a 460.000 toneladas (HIRANO, 2003a).

Analisando-se a área e a produção brasileira dos últimos dez anos, verificou-se que houve um decréscimo de $9 \%$ na área cultivada, enquanto que, a produção aumentou $8 \%$, significando um ganho em produtividade. A produção paranaense, na safra 2002/ 03, foi de 609.000 toneladas em uma área plantada de 30.000 ha (SEAB - DERAL, 2004).

O Estado do Paraná já chegou a contribuir com $27 \%$ da produção nacional mas, na ultima década observou-se uma redução da área cultivada devido a problemas agronômicos, o que coincidiu com o aumento da produção no Estado de Minas Gerais (GODOY, 2000).

O Estado de Santa Catarina é o quinto produtor nacional de batata para consumo e um importante produtor de batata-semente (INSTITUTOCEPA / 2001). O Estado apresenta ótimas condições climáticas para o desenvolvimento e a produção da batata, sendo possível o cultivo o ano todo (SOUZA et al., 1999).

O tubérculo de batata-semente, imediatamente após ser colhido, não inicia o processo de brotação, mesmo quando colocado em condições de temperatura e umidade para que isto ocorra, devido a uma condição endógena denominada dormência que é regulada pelo balanço hormonal entre promotores e inibidores do crescimento (HEMBERG, 1985).

Nos países de clima tropical, onde há condições de plantio de pelo menos duas safras durante o ano, são recomendados cultivares com período de dormência curto com o objetivo de se obter batatas-semente brotadas mais rapidamente após o plantio. Para as cultivares que não possuem esta característica, foram desenvolvidas tecnologias de forçamento artificial para quebrar a dormência e a dominância apical da batata-semente (HIRANO, 2003a).

O emprego de diversos métodos físicos e químicos tem apresentado eficiência no rompimento de dormência das gemas do tubérculo. Um dos métodos físicos utilizados é o do abafamento dos tubérculos que estimula a brotação, devido ao aumento da temperatura, diminuição da concentração de oxigênio e aumento da concentração de gás carbônico (SCHILTE, 1990). Existem produtos químicos que aceleram a brotação, sendo um deles o dissulfeto de carbono, que vem mostrando resultados satisfatórios na quebra da dormência acelerando a brotação.

O gás dissulfeto de carbono pode possibilitar antecipação do plantio apesar das variações nas respostas obtidas pelos pesquisadores (DANIELS et al., 1982). Estes autores observaram que houve uma correlação positiva com o número de hastes e a produção de tubérculos.

\section{METODOLOGIA}

Experimento foi conduzido no Laboratório de Análise e Tecnologia de Sementes do Departamento de Fitotecnia e Fitossanitarismo do Setor de Ciências Agrárias da UFPR. Para todas as cultivares foram utilizados tubérculos de batata-semente do tipo II (entre 41 e $50 \mathrm{~mm}$ de diâmetro) (BRASIL, 1988). O trabalho de pesquisa foi dividido em dois experimentos: $O$ primeiro experimento foi realizado outubro de 2003, utilizando as cultivares Asterix e Cupido, oriundas da região de Guarapuava (PR), o segundo experimento foi realizado em Fevereiro de 2004, com as cultivares Asterix e Monalisa, oriundas da região de Canoinhas (SC). Nos dois experimentos a aplicação do gás se deu após 30 dias da colheita.

Os tubérculos de batata-semente de todas as cultivares utilizadas foram acondicionadas em caixas de papelão medindo $54 \times 32 \times 40 \mathrm{~cm}$, totalizando um volume de $69,120 \mathrm{~cm}^{3}$, onde foram aplicados os seguintes tratamentos: exposição das batatassemente com o gás de dissulfeto de carbono nas doses de $15 \mathrm{cc} ; 20 \mathrm{cc} ; 25 \mathrm{cc} ; 30 \mathrm{cc} ; 35 \mathrm{cc}$ por $\mathrm{cm}^{3}$, as quais foram transformadas de acordo com o volume das caixas de papelão, em: 1,04; 1,38; 1,73; 2,07; e $2,42 \mathrm{ml} \mathrm{cm}^{-3}$ respectivamente, durante os períodos de 60,72 e 84 horas. Após tais períodos as caixas foram abertas e os tubérculos ficaram expostos às condições ambiente. O gás de dissulfeto de carbono foi colocado em um recipiente plástico de $250 \mathrm{ml}$ amarrado na parte interna e superior da caixa de papelão. O líquido do dissulfeto de carbono foi colocado nos recipientes plásticos com o auxilio de uma pipeta nas cinco dosagens correspondentes. Em seguida a caixa foi vedada com fita adesiva e colocada dentro de um saco plástico de polietileno, que foi amarrado com barbante se ajustando à caixa, para evitar a dispersão do gás.

As caixas foram colocadas sobre as bancadas do laboratório, mantidas em temperatura ambiente, sob luz indireta para evitar o esverdeamento dos tubérculos e a produção de brotos estiolados, a temperatura ambiente manteve-se entre 22 a $25 \stackrel{\circ}{\circ}$, durante os 30 dias do experimento.

O período de 30 dias de avaliação correspondem à aplicação do gás após os períodos de exposição as caixas foram abertas, permanecendo 7 dias sem leitura. A primeira leitura foi realizada no $14^{\circ}$ e $15^{\circ}$ dia após a abertura das caixas, a segunda leitura $21^{\circ}$ e $22^{\circ}$ e, a terceira e última leitura $29^{\circ}$ e $30^{\circ}$ dias após a abertura das caixas para todas as cultivares utilizadas nos experimentos. 
Os tubérculos foram avaliados um a um, recebendo uma marcação de 1 a 20 para evitar troca de tubérculos nas avaliações seguintes. Nas avaliações foram observados os números de brotos emitidos pelas gemas e o comprimento destes brotos, estes medidos por um paquímetro.

O delineamento experimental foi inteiramente casualizado, para os dois experimentos, em esquema fatorial $(3 \times 5)$ três tempos $(60,72$ e 84 horas) e cinco doses $\left(1,04 ; 1,38 ; 1,73 ; 2,07\right.$; e $\left.2,42 \mathrm{ml}^{-\mathrm{cm}^{-3}}\right)$, com quatro repetições. A separação das médias foi determinada pelo DMS (0,01\%).

\section{RESULTADOS E DISCUSSÃO}

Os resultados obtidos na Tabela 1 , demonstraram que, para a variável número de brotos, a exposição dos tubérculos de batata-semente ao gás dissulfeto de carbono no tempo de 72 horas $(43,40)$, proporcionaram maior número de brotos $\mathrm{e}$ diferenciando-se significativamente dos demais tratamentos. Para a variável comprimento de brotos os tempo de 72 e 84 horas $(6,37 ; 6,51 \mathrm{~cm}$ respectivamente) não mostraram diferenças estatística entre si, mas foram superiores significativamente quando comparado com o resultado obtido para o tempo de exposição de 60 horas.

A interação entre doses e tempo (Tabela 2), de exposição dos tubérculos ao gás dissulfeto de carbono, foi altamente significativo para o tempo de 72 horas utilizando as dosagens $1,73 \mathrm{ml} . \mathrm{cm}^{-3}$; $2,07 \mathrm{ml}^{\mathrm{cm}}{ }^{-3}(55,75 ; 56,50 \mathrm{~cm}$ respectivamente) não diferenciando-se da dosagem de 2,42 $\mathrm{ml} \mathrm{cm}^{-3}$ $(45,25 \mathrm{~cm})$. Nos tempos de exposição de 60 e 84 horas os resultados mostraram que não houve diferença significativa entre as dosagens utilizadas nos tratamentos.

TABELA 1 - Número e comprimento de brotos da cultivar Asterix, (Experimento 01) de tubérculos expostos ao gás dissulfeto de carbono em diferentes tempos. Outubro, 2003.

\begin{tabular}{ccc}
\hline Tempo & № de Brotos & Comprimento de Brotos $(\mathrm{cm})$ \\
\hline 60 & $26,65 \mathrm{~b}$ & $5,07 \mathrm{~b}$ \\
72 & $43,40 \mathrm{a}$ & $6,37 \mathrm{a}$ \\
84 & $26,60 \mathrm{~b}$ & $6,51 \mathrm{a}$ \\
\hline CV\% & 12,84 & 25,95 \\
\hline
\end{tabular}

Médias seguidas pela mesma letra nas colunas não diferem significativamente entre si a $0,01 \%$ de probabilidade pelo teste de DMS.

TABELA 2 - Número de brotos da cultivar Asterix (Experimento 01), tubérculos expostos em três diferentes tempos e cinco dosagens do gás dissulfeto de carbono. Outubro, 2003.

\begin{tabular}{lccc}
\hline \multirow{2}{*}{ Dosagens ml.cm } & \multicolumn{3}{c}{ Tempo (h) } \\
\cline { 2 - 4 } & 60 & 72 & 84 \\
\hline 1,04 & $26,50 \mathrm{a}$ & $24,50 \mathrm{c}$ & $26,25 \mathrm{a}$ \\
1,38 & $31,25 \mathrm{a}$ & $35,00 \mathrm{bc}$ & $32,25 \mathrm{a}$ \\
1,73 & $28,50 \mathrm{a}$ & $55,75 \mathrm{a}$ & $24,00 \mathrm{a}$ \\
2,07 & $24,50 \mathrm{a}$ & $56,50 \mathrm{a}$ & $23,25 \mathrm{a}$ \\
2,42 & $22,50 \mathrm{a}$ & $45,25 \mathrm{ab}$ & $27,25 \mathrm{a}$ \\
\hline
\end{tabular}

C.V. $=25,93 \%$

Médias seguidas pela mesma letra nas colunas não diferem significativamente entre si a 0,01\% de probabilidade pelo teste de DMS

Observando-se os resultados obtidos para a cultivar Cupido (Experimento 01), na Tabela 3 as médias demonstraram que, para a variável número de brotos, a exposição dos tubérculos ao gás dissulfeto de carbono no tempo de 72 horas $(23,30)$ foi o melhor resultado, com uma diferença altamente significativa dos demais. Para a variável comprimento de brotos os tempos de 72 e 84 horas $(4,54 ; 4,46 \mathrm{~cm}$ respectivamente), não mostram diferença estatística entre si, sendo altamente significativos quando analisando os resultados obtidos para o tempo de exposição de 60 horas .

Analisando a Tabela 4 os resultados obtidos, para a variável número de brotos, verificou-se que a interação entre doses e tempo de exposição dos tubérculos ao gás dissulfeto de carbono, foi altamente significativo. Para o tempo de 72 horas utilizando a dosagem de $1,73 \mathrm{ml}_{\text {. }} \mathrm{cm}^{-3}(27,5)$, está produziu um número de brotos maior que as outras dosagens usadas apesar de não haver diferença estatística entre elas. No tempo de exposição de 60 horas os resultados mostraram que não ocorreu diferença significativa entre as dosagens utilizadas nos tratamentos. Para o tempo de 84 horas de exposição os resultados mostram que a melhor dosagem foi a de $1,04 \mathrm{ml}_{\mathrm{cm}}{ }^{-3}$, com um número de brotos bem superior às outras dosagens $(21,00)$. Ainda assim o tempo de 72 horas mostrou uma melhor produção de brotos. 
KOWALSKI, S.M.O. Gás dissulfeto de carbono na...

TABELA 3 - Número e comprimento de brotos da variedade Cupido (Experimento 01). Tubérculos expostos ao gás dissulfeto de carbono em diferentes tempos. Outubro, 2003.

\begin{tabular}{ccc}
\hline Tempo & № de Brotos & Comprimento de Brotos $(\mathrm{cm})$ \\
\hline 60 & $16,90 \mathrm{~b}$ & $3,90 \mathrm{~b}$ \\
72 & $23,30 \mathrm{a}$ & $4,54 \mathrm{a}$ \\
84 & $15,27 \mathrm{~b}$ & $4,46 \mathrm{a}$ \\
\hline C.V. & $14,31 \%$ & $11,25 \%$ \\
\hline
\end{tabular}

Médias seguidas pela mesma letra nas colunas não diferem significativamente entre si a 0,01\% de probabilidade pelo teste de DMS

TABELA 4 - Número de brotos da cultivar Cupido (Experimento 01) tubérculos expostos em três diferentes tempos e cinco dosagens do gás dissulfeto de carbono. Outubro, 2003.

\begin{tabular}{lcll}
\hline \multirow{2}{*}{ Dosagens ml.cm } & \multicolumn{3}{l}{ Tempo $(\mathrm{h})$} \\
\cline { 2 - 4 } & 60 & $22,75 \mathrm{ab}$ & 84 \\
\hline 1,04 & $17,75 \mathrm{a}$ & $22,75 \mathrm{ab}$ & $21,00 \mathrm{a}$ \\
1,38 & $15,75 \mathrm{a}$ & $27,50 \mathrm{a}$ & $14,50 \mathrm{bc}$ \\
1,73 & $18,50 \mathrm{a}$ & $22,50 \mathrm{ab}$ & $15,75 \mathrm{~b}$ \\
2,07 & $18,75 \mathrm{a}$ & $21,00 \mathrm{~b}$ & $14,50 \mathrm{bc}$ \\
2,42 & $13,75 \mathrm{a}$ & & $10,63 \mathrm{c}$ \\
\hline
\end{tabular}

C.V. $=14,31 \%$

Médias seguidas pela mesma letra nas colunas não diferem significativamente entre si a 0,01\% de probabilidade pelo teste de DMS

Observou-se que os resultados das análises estatísticas para o (Experimento 02) cultivares Asterix e Monalisa, observou-se que ocorreu uma diferença altamente significativa entre os tratamentos para o fator tempo para a variável comprimento de brotos e uma diferença significativa para a variável número de brotos.

A interação entre tempo $x$ dosagem utilizadas nos tratamentos e o fator dosagem não mostraram resultados significativos.
A Tabela 5 cultivar Asterix (Experimento 02), mostra que os tubérculos expostos ao gás dissulfeto de carbono no tempo de 72 horas produziram um número de brotos maior que os demais tempos de exposição $(50,05)$, apesar de não haver diferença estatística entre o tempo de 84 horas $(46,75)$. Para a variável comprimento de brotos, os resultados foram altamente significativos quando os tubérculos foram expostos ao gás dissulfeto de carbono.

TABELA 5 - Número e comprimento de brotos da cultivar Asterix (Experimento 02) tubérculos expostos ao gás dissulfeto de carbono em diferentes tempos. Fevereiro, 2004.

\begin{tabular}{ccc}
\hline Tempo & № de Brotos & Comprimento de Brotos $(\mathrm{cm})$ \\
\hline 60 & $43,35 \mathrm{~b}$ & $6,09 \mathrm{c}$ \\
72 & $50,05 \mathrm{a}$ & $6,77 \mathrm{~b}$ \\
84 & $46,75 \mathrm{ab}$ & $7,78 \mathrm{a}$ \\
\hline C.V. & $15,94 \%$ & $8,48 \%$ \\
\hline
\end{tabular}

Médias seguidas pela mesma letra nas colunas não diferem significativamente entre si a 0,01\% de probabilidade pelo teste de DMS

Na Tabela 6 observa-se que, para a variável número de brotos, a exposição dos tubérculos ao gás dissulfeto de carbono no tempo de 72 horas mostraram um resultado altamente significativo, produzindo uma quantidade de brotos bem superior
$(44,15)$. Para a variável comprimento de brotos, os resultados mostraram que para os tempos de exposição de $72(5,98 \mathrm{~cm})$ e $84(5,71 \mathrm{~cm})$ horas não diferem estatísticamente, porém, o tempo de 72 horas produziu brotos de maior comprimento. 
KOWALSKI, S.M.O. Gás dissulfeto de carbono na...

TABELA 6 - Número e comprimento de brotos da variedade Monalisa (Experimento 02) tubérculos expostos ao gás dissulfeto de carbono em diferentes tempos. Fevereiro, 2004.

\begin{tabular}{ccc}
\hline Tempo & № de Brotos & Comprimento de Brotos (cm) \\
\hline 60 & $39,70 \mathrm{~b}$ & $5,71 \mathrm{ab}$ \\
72 & $44,15 \mathrm{a}$ & $5,98 \mathrm{a}$ \\
84 & $39,95 \mathrm{~b}$ & $5,44 \mathrm{~b}$ \\
\hline C.V. & $14,56 \%$ & $9,25 \%$ \\
\hline
\end{tabular}

Médias seguidas pela mesma letra nas colunas não diferem significativamente entre si a $0,01 \%$ de probabilidade pelo teste de DMS

As dosagens para este experimento não foram possíveis de serem determinadas devido aos resultados não serem significativos.

\section{CONCLUSÕES}

Experimento 01 - Os resultados sugerem que para as cultivares Asterix e Cupido que a exposição dos tubérculos ao gás dissulfeto de carbono no tempo de 72 horas com a utilização da dosagem $1,73 \mathrm{ml}_{\mathrm{cm}} \mathrm{cm}^{-3}$, equivalente a $25 \mathrm{cc} \mathrm{m}^{-3} \mathrm{de}$ quebra a dormência das gemas.
Experimento 02 - Para as cultivares Asterix e Cupido, o resultado sugerem que o uso do gás dissulfeto de carbono foi eficaz para a quebra da dormência das gemas dos tubérculos de batatasemente, quando expostas no tempo de exposição de 72 horas, utilizando as dosagens entre 1,04 à $2,07 \mathrm{ml}_{\mathrm{cm}}^{-3},\left(15\right.$ a $\left.16 \mathrm{cc} \mathrm{m}^{-3}\right)$.

\section{REFERÊNCIAS}

1. GODOY, R.C.B. Prognóstico da cultura de batata - safra 2000-01: Acompanhamento da Situação Agropecuária do Paraná. Curitiba: SEAB, 2000. 26 v. 10 n. 14-32 p.

2. HEMBERG. T. Potato rest. In: L. I.; P. H. Potato Physiology. Orlano: Academic Press, 1985. 353-388 p.

3. HIRANO, E. Batata-semente, Básica, Registrada e Certificada. PEREIRAA. S.; DANIELS, J. (Ed.). In: 0 cultivo de batata na região sul do Brasil. Brasilia: Embrapa informações tecnológicas, 2003a. 475-494 p.

4. INSTITUTO CEPA. Síntese anual da agricultura de Santa Catarina. Florianópolis (SC.), 2001. 248 p.

5. SEAB-DERAL. BATATA: aspectos econômicos. Curitiba (PR.), 2004. Disponível em: <http;/www.pr.gov.br/seab/credencial.> acesso em 25 março de 2005.

6. SCHOLTE, K. Breaking dormancy of seed potatoes. Wageningen: International Agricultural Center, $1990.4 \mathrm{p}$.

7. SOUZA,Z. S.; SILVA, A C.F.; BEPPLER N. R. Cadeias produtivas do Estado de Santa Catarina. Florianópolis (SC.), 1999. $24 \mathrm{p}$. 